\title{
Sero-Prevalence of HIV and Syphilis Infection Among Pregnant Mothers Attended Labor Ward at Wolaita Sodo University Teaching Referral Hospital: A Five-Year Retrospective Study (2012-2016)
}

\author{
Temesgen Anjulo Ageru ${ }^{1, *}$, Temesgen Lera Abiso ${ }^{2, *}$ \\ ${ }^{1}$ Department of Medical Laboratory, Wolaita Sodo University Teaching and Referral Hospital, Wolaita Sodo, Ethiopia \\ ${ }^{2}$ School of Public Health, College of Health Sciences and Medicine, Wolaita Sodo University, Wolaita Sodo, Ethiopia
}

Email address:

teanjulo@gmail.com (T. A. Ageru), temelera@gmail.com(T. L. Abiso)

${ }^{*}$ Corresponding author

\section{To cite this article:}

Temesgen Anjulo Ageru, Temesgen Lera Abiso. Sero-Prevalence of HIV and Syphilis Infection Among Pregnant Mothers Attended Labor Ward at Wolaita Sodo University Teaching Referral Hospital: A Five-Year Retrospective Study (2012-2016). American Journal of Health Research. Vol. 6, No. 3, 2018, pp. 67-73. doi: 10.11648/j.ajhr.20180603.11

Received: March 29, 2018; Accepted: May 31, 2018; Published: August 14, 2018

\begin{abstract}
Sexually transmitted infections (STIs) such as HIV and Syphilis infections are the major public health problem in the developing countries. The prevalence of syphilis and HIV infections among pregnant women has been shown to vary by geographic areas, study population and time-period. Objective: The aim of study was to assess sero prevalence of HIV and syphilis and associated factors among pregnant women attended labor ward at Wolaita Sodo University Teaching and Referral Hospital. Method: Retrospective study was conducted using standard extraction format to assess the sero-prevalence of HIV and syphilis among pregnant women attending labor ward of WSTRH. Data were analyzed by using SPSS version 20.0 software and result was summarized using means and percentages and presented by using figure and tables. Descriptive statistics were used. Result: Overall prevalence of sexually transmitted infection among pregnant women in labor was $7.4 \%$ from these HIV sero-prevalence accounts for 3.8\% and VDRL positivity enumerates to be 3.7\%. HIV-syphilis co-infection was found to be $0.9 \%$. Higher sero-prevalence of HIV, syphilis and co-infection rate was reported in 2016, among age category of 35-44 years. Conclusion and recommendation: Significant percentage of HIV, syphilis sero-prevalence and co-infection rate was found in this study with increased proportion in 2016. Strong antenatal screening and appropriate strategies should be devised for prevention and control of HIV, syphilis, in women of reproductive age.
\end{abstract}

Keywords: HIV, Syphilis, Co-Infection, Sero-Prevalence, Pregnant Women

\section{Introduction}

Sexually transmitted infections (STIs) are a group of infectious or communicable diseases in which their primary mode of transmission is through sexual contact and they are among the major causes of illnesses throughout the world particularly in the developing countries. They are extraordinarily common with an estimated 340 million new cases of "curable" infections (including syphilis) occurring each year worldwide in men and women aged 15-49 years [1]. The impact of STIs is particularly severe for women of reproductive age, since such infections have few symptoms and may go untreated until serious problems develop [2].

Human immunodeficiency virus (HIV) is an RNA virus that belongs to a family of viruses known as retroviruses and causes acquired immunodeficiency syndrome (AIDS). The virus is a highly variable which mutates very readily meaning it have many different strains, even within the body of a single infected person [3]. HIV infects vital cells in the human immune system such as helper $\mathrm{T}$ cells (specifically $\mathrm{CD} 4+\mathrm{T}$ cells) and its infection leads to low levels of CD4+ T cells through different mechanisms [4].

Syphilis is one of the sexually transmitted infections and is a reportable disease caused by Treponema pallidum. The 
bacteria can survive only briefly outside of the body; thus, transmission almost always requires direct contact with the infectious lesion through acquired or congenital [5].

Syphilis is transmitted in utero by trans-placental passage of the Treponema. The risk of transmission is related to the maternal Treponema load; hence early maternal infection carries a much greater risk of transmission than late infection. Indeed, almost all babies born to mothers with untreated primary or secondary infection will be infected, but the likelihood of transmission and the severity of infection will fall with subsequent pregnancies as the mother's Treponema load will fall with time [6]. Syphilis and HIV co-infection is now increasingly common all over the world. One of the major concerns regarding the coexistence of HIV and syphilis is that syphilis, as other genital ulcer diseases, might facilitate HIV acquisition and transmission due to interfering with the natural mucosal and epithelial barriers and by causing local inflammation. Furthermore, syphilis has been found to decrease (at least transiently) CD4 T-cell counts and increase plasma viral load in patients chronically infected with HIV where both of which have been linked to increase in HIV transmission [7].

Early diagnosis and treatment of pregnant women who tested positive for syphilis have been shown to be effective in reducing still-birth, neonatal death and congenital infection by more than $55 \%[6,8]$. Importantly, these interventions have been estimated to be highly cost-effective even in low in-come countries [6].

The prevalence of syphilis and HIV infections has been shown to vary by geographic areas, study population and time-period [9-12]. Thus, obtaining periodical estimates of the local epidemiological picture of these infections by monitoring various risk groups is essential for guiding clinical action, resource allocation, and intervention protocols to make progress toward elimination.

\section{Methods and Materials}

\subsection{Study Setting}

The study was conducted at Wolaita Sodo University Teaching Referral Hospital which is situated at southern of Sodo town, Wolaita Zone, $396 \mathrm{~km}$ from Addis Ababa, Ethiopia. It is serving people in catchment's area of 2 million people including neighboring Dawuro zone, Gamogofa zone, and Kambata Tambaro one. The hospital organized in department, case-teams and working hours are according to BPR standard. There are seven case teams, OPD, Emergency, Inpatient, Delivery, Dentistry and Ophthalmology. The hospital has 200 beds for inpatient service which is on medical, pediatrics, surgical gynecology and obstetrics ward. 2.2. Study Design

A retrospective study was conducted to assess the seroprevalence of syphilis and HIV among pregnant women attended labor ward from 30/09/2012 to 30/09/2016.

\subsection{Population}

\subsubsection{Source Population}

The source population were all pregnant women who attended labor ward in Wolaita Sodo University teaching referral hospital from 30, June 2012-30, June 2016.

\subsubsection{Study Population}

The study populations were all pregnant women who attended labor ward in WSUTRH from 2012 up to 2016 and fulfilling the inclusion criteria.

\subsection{Inclusion and Exclusion Criteria}

\subsubsection{Inclusion Criteria}

All pregnant mothers who attended and registered in the document during the study period.

\subsubsection{Exclusion Criteria}

All pregnant mothers with incomplete information (especially age, HIV \& Syphilis status.)

Sampling Techniques:

Convenient data collection technique according to inclusive and exclusive criteria (2012-2016).

\subsection{Data Collection Procedures}

Demographic data was collected based on our inclusion and exclusion criteria from the record using well-structured record format (annex ii) from antenatal care clinical records. Laboratory data of sero-positivity of syphilis was obtained from Wolaita Sodo teaching and referral University hospital laboratory

\subsection{Quality Assurance}

The data was checked for consistency and completed registration or recording document.

\subsection{Ethical Considerations}

The ethical clearance was obtained from the Ethical review committee of Wolaita Sodo University, college of health science and medicine. Formal permission was also obtained from Wolaita Sodo University Referral Hospital. For reasons of privacy, all data were kept confidential. Anonymity of records was maintained by using client ANC registration number and unique code numbers used by service providers at Wolaita Sodo University teaching referral hospital.

\subsection{Data Processing and Analysis}

The preliminary data were checked and processed depending on the variables. Analyzed using SPSS Version-20 software and results were summarized using means and percentages andpresented by using graphs and tables.

\section{Result}

\subsection{Socio Demographic Characteristics}

A total number of 4166 women were attended labor ward 
in 2012-2016 in Wolaita Sodo university teaching referral hospital (WSUTRH). Four thousand twenty two women (4022) were included the study with complete information that is important for the study. The majority, $62 \%$ was found in the age group of 25-34 whereas the least abundant one was found in the age category of 35-44 years old. Table 1

Table 1. Socio-demographic characteristics of pregnant mothers in the antenatal care unit of WSUTRH from 2012-2016.

\begin{tabular}{lll}
\hline Age & Number & Percent $(\%)$ \\
\hline $15-24$ & 1333 & 33.1 \\
$25-34$ & 2492 & 62 \\
$35-44$ & 197 & 4.9 \\
\hline
\end{tabular}

\subsection{Sero-Prevalence of HIV among Pregnant Mothers}

Overall prevalence of 299/4022(7.4\%) mothers were found for either one of HIV or syphilis. HIV sero-positive prevalence of $3.8 \%$ were identified in this study of which the largest percentage, $13.2 \%$ were recorded in year 2016 . On the other hand, the minimum percentage was shown in year 2013 with magnitude of $2.1 \%$. The overall trend of HIV seropositivity was depicted irregular feature. Table 2

Table 2. HIV sero-prevalence among pregnant women followed antenatal care in WSUTRH from 2012-2016.

\begin{tabular}{llll}
\hline \multicolumn{5}{l}{ HIV sero-prevalence } \\
\hline Year & Positive & Negative & Total \\
\hline 2012 & $39(3.3)$ & $1143(96.7)$ & $1182(29.4)$ \\
2013 & $28(4.2)$ & $641(95.8)$ & $669(16.6)$ \\
2014 & $20(2.1)$ & $919(97.9)$ & $939(23.3)$ \\
2015 & $22(2.1)$ & $1006(97.9)$ & $1028(25.6)$ \\
2016 & $42(20.6)$ & $162(79.4)$ & $204(5.1)$ \\
Total & $151(3.8)$ & $3871(96.2)$ & $4022(100)$ \\
\hline
\end{tabular}

\subsection{Sero-Prevalence of Syphilis among Pregnant Mothers}

An overall prevalence of Treponema pallidum, 3.7\% was detected among pregnant mothers with the highest seroprevalence noted in 2016 and the least percentage were found in years 2012 and 2013. Syphilis detection rate was shown an irregular trend across the years. Table 3

Table 3. Syphilis sero-prevalence among pregnant women attending antenatal care in WSUTRH from 2012-2016.

\begin{tabular}{llll}
\hline \multirow{2}{*}{ Year } & \multicolumn{4}{l}{ Syphilis sero-prevalence } \\
\cline { 2 - 4 } & Positive & Negative & Total \\
\hline 2012 & $49(4.3)$ & $1133(95.7)$ & $1182(29.4)$ \\
2013 & $14(2.1)$ & $655(97.9)$ & $669(16.6)$ \\
2014 & $23(2.4)$ & $916(97.6)$ & $939(23.3)$ \\
2015 & $34(3.3)$ & $994(96.7)$ & $1028(25.6)$ \\
2016 & $27(13.2)$ & $177(86.8)$ & $204(5.1)$ \\
Total & $147(3.7)$ & $3875(96.3)$ & $4022(100)$ \\
\hline
\end{tabular}

\subsection{HIV-Syphilis Co-Infection, among Women Attended Labor Ward}

From the total women included in the study $0.94 \%$ syphilis-HIV co-infection was observed. Eight seven
87(57.6\%) women were positive for syphilis among HIV positive cases. Linear increasing trend of syphilis prevalence were found in the five years. The overall prevalence of sexually transmitted infections (HIV and syphilis) were highest in year $2016(28.9 \%)$ but the least abundant were noticed in 2014(4.4\%). Irregular feature (decreasing and increasing) trends were found for overall infection among women who attended labor ward in WSUTRH. Table 4

Table 4. Syphilis-HIV co-infection among pregnant women attending antenatal care in WSUTRH from 2012-2016.

\begin{tabular}{llll}
\hline \multicolumn{4}{l}{ Syphilis-HIV co-infection } \\
\hline Year & Positive & Negative & HIV/syphilis** \\
2012 & $7(0.6)$ & $1175(99.4)$ & $88(7.44)$ \\
2013 & $4(0.6)$ & $665(99.4)$ & $42(6.3)$ \\
2014 & $6(0.64)$ & $933(99.4)$ & $43(4.4)$ \\
2015 & $10(1)$ & $1018(99)$ & $56(5.4)$ \\
2016 & $11(5.4)$ & $183(94.6)$ & $59(28.9)$ \\
Total & $38(0.9)$ & $3984(99)$ & $298(7.4)$ \\
\hline
\end{tabular}

** Sero-positive pregnant women for either HIV or syphilis

\subsection{Age among Sero-Positive Women}

Sero-prevalence of HIV, Syphilis and HIV-syphilis coinfection was higher, $8.1 \%, 10.4 \%$ and $4.1 \%$ respectively among age groups from 35-44. The least abundant seropositivity were found in age category of 25-34 for HIV (3.3\%), syphilis $(2.8 \%)$ and co-infection $(0.7 \%)$. Table 5

Table 5. Socio-demographic variables among HIV and syphilis sero-positive pregnant mothers at WSUTRH.

\begin{tabular}{llll}
\hline Age & HIV No (\%) & Syphilis No (\%) & $\begin{array}{l}\text { Co-infection No } \\
(\%)\end{array}$ \\
\hline $15-24$ & $53(4 \%)$ & $57(4.3)$ & $13(1)$ \\
$25-34$ & $82(3.3)$ & $69(2.8)$ & $17(0.7)$ \\
$35-44$ & $16(8.1)$ & $21(10.7)$ & $8(4.1)$ \\
\hline
\end{tabular}

\section{Discussion}

This study revealed that the sero-prevalence of HIV and Syphilis infections and the co-infection of HIV and syphilis rate among women attending labor ward at Wolaita Sodo University Teaching Referral Hospital, Sodo, Ethiopia. All women attending labor ward have to be screened for HIV and Syphilis at labor and delivery if they are not tested at ANC follow-up.

This study revealed that sero-prevalence of HIV among pregnant women attending labor ward is $3.7 \%$. This finding is in line with study reported in Nigeria, 3.8\% Niger, $4.1 \%$ and Cameroon $4.2 \%[13,33,34]$. In the contrary, our finding is lower than $6.6 \%$ sero-positivity in Cameroon[35], 7.6\% in Northern Tanzania [36] and $8 \%$ in Brazil [37] and 14\% in South Africa [38], and. HIV sero-prevalence in the current study is lower than previous studies conducted in Ethiopia with magnitude of $5.2 \%$ [39], 7.7\% [40], 9.6\%[41], $11.2 \%$ [42], 10.33\% [43].

On the other hand, lower findings as compared with our study were also reported in previous literatures of $0.38 \%$ in India [44], 1.8\% in South Ethiopia [45]. Moreover, the 
finding of the present study was also higher than to the adult $(1.3 \%)$ and the women national HIV prevalence of Ethiopia (1.8\%).

The variation of this finding compared to the national HIV prevalence may be as result of difference in sample size, study area coverage and study population. Where; the national prevalence done on the total population and covers all over the nation whereas our finding has focused only in one segment of the population and limited to one study area. But compared to the study conducted in rural hospitals of southern Ethiopia the variation may be due to difference in living conditions where living condition of urban people are more crowded than rural setting this facilitates the risk of HIV transmission.

The higher prevalence of HIV in studies conducted in Northern Ethiopia, Gondar may be due to socio-cultural difference where early marriage and tattooing can be considered as a main contributor for the variation. As compared with studies conducted in Addis Ababa, our finding was much lower. This could be attributed to the overcrowding living status of urban areas and access to sexual exposure risky behaviors are more common in urban areas than this study where these factors increase the transmissibility of HIV.

Syphilis remains a major cause of morbidity and mortality in the world despite the availability of effective treatment. Interventions including early $\mathrm{ANC}$, massive screening and prompt treatment with antibiotics have been reported to reduce syphilis attributable-risks [46]. Sero-prevalence of syphilis in the current study was $3.7 \%$, which was consistent with studies conducted in Gondar, 3.7\% in Gondar [43], 3\% in Madagascar [47], Brazil [31] Bangladesh [48] each, and $2.9 \%$ in Addis Ababa, Ethiopia [49].

In contrast, it was higher than $2.3 \%$ sero-prevalence in the study done in Gondar health centers [32], 1.6\% in Tanzania [50], $1 \%$ in Gondar University Teaching Hospital [9]. However, it was lower than previous studies conducted in Northwest Ethiopia with magnitude of $13.7 \%$ [51] and $10 \%$ in Southwestern Nigeria [52].

The variation might be the result of differences in culture, socioeconomic status, prevention and control measures, risk factors, level of awareness, and methods used for diagnosis where previous cross-sectional studies used syphilis specific tests like Treponema pallidum hemaglutinnin antigens and fluorescent treponemal antibodies which can detect syphilis at early stage and after treatment. The lower prevalence of syphilis among the study population could be attributed to the increase in syphilis screening at the ANC and effective treatment with antibiotics in the last 10 years.

Syphilis has also acquired a new potential for morbidity and mortality through association with increased risk of HIV infection. Because of their common route of transmission as well as the fact that they are mainly blood borne infections, syphilis and HIV co-infection continues to be a public health problem especially in the low income settings. More specifically, syphilis causes genital ulcer and facilitates HIV entry and shading. Besides, it induces immune activation and favor viral replication, which in turn accelerate HIV transmissibility [53].

The rate of HIV and syphilis co-infection varies depending on the prevalence of both infections in the community or the patient group being studied, along with individual risk factors. HIV-syphilis co-infection, $0.9 \%$ in the present study is in harmony with a study conducted in Gondar, 1\% [43] but higher than $0.3 \%$ in Tanzania [50] but lower than $2.2 \%$ reported in north Ethiopia [9]. In the contrary to this, our finding was higher than, $0.05 \%$ reported in Nigeria [53].

Strength of the study

i. This study included a five year data with retrospective design so it could show the progress and weakness of the prevention policy

ii. This is the first study conducted in WSUTRH so it could serve as a baseline study for future works

Limitation of a study

i. The use of only serological tests for the diagnosis of HIV and syphilis, which may lead to false positivity and negativity and, ultimately, underestimate or overestimate of the results of our study.

ii. The study is pure descriptive so we can't analyze associated factors with sero-prevalence of HIV and syphilis.

\section{Conclusion}

This study showed significant proportion of HIV and syphilis sero-prevalence were detected, indicating that they are significant public health problems. Both HIV and syphilis revealed the highest percentage in 2016 this could show the trend is increasing across the years.

\section{Recommendations}

Based on the findings the following recommendations are given:

i. Further studies on syphilis and HIV that includes different study sites are recommended

ii. Health education about identified risk factors for HIVHBV and prevention methods should be given to expectant mothers.

iii. There should be a need to enhance antenatal screening to reduce and ultimately prevent vertical and horizontal transmission of HIV and syphilis.

iv. Likewise, appropriate strategies should be devised for prevention and control of HIV, syphilis, in women of reproductive age.

v. Moreover, societal factors that contribute to HIV and syphilis transmission need to be further studied in the general population, which will be helpful to prevent future HIV and syphilis transmission in the general population.

\section{Competing Interests}

The authors have declared that no competing interests exist. 


\section{Acronyms and Abbreviations}

$\begin{array}{ll}\text { AIDS } & \text { Acquired Immunodeficiency Syndrome } \\ \text { ANC } & \text { Antenatal care } \\ \text { HIV } & \text { Human Immunodeficiency Virus } \\ \text { OR } & \text { Odds ratio } \\ \text { MDG } & \text { Millennium Development Goal } \\ \text { SPSS } & \text { Statistical Package for Social Science } \\ \text { RPR } & \text { Rapid Plasma Reagin } \\ \text { STI } & \text { Sexually Transmitted Infection } \\ \text { VDRL } & \text { Venereal Disease Research Laboratory } \\ \text { WHO } & \text { World Health Organization } \\ \text { WSUTRH } & \text { Wolaita Sodo University Teaching Referral } \\ & \text { Hospital }\end{array}$

\section{Acknowledgements}

We would like to Wolaita Sodo University Teaching and Referral Hospital, department of medical laboratory office for assigning us to Wolaita Sodo university teaching referral hospital and giving us the opportunity to study five years retrospective data analysis. We also thank the administrative bodies, laboratory department and labor and delivery unit of WSUTRH for their cooperative willingness to provide recorded patient information

\section{Availability of Data \& Materials}

The data for this research is available, so we can contact you when you need our data for future process.

\section{Authors' Contribution}

Temesgen Anjulo Ageru: Developed design, participated in the statistical analysis, developed the sequence alignment and drafted the manuscript.

Temesgen Lera Abiso: Performed the statistical analysis, Participated in the design of the study, participated in the draft of manuscript, Participated in the sequence alignment

These authors read and approved the manuscript.

\section{References}

[1] Aron G, Laura B, Kelly C, Stephen K. Epidemiology of Sexually Transmitted Infections. Sex Trans Infectand Sex Transm Dis.2011; 13-23.

[2] Frank N, Okonko I, Okerentugba P, Jaja N. Detection of HIV1/2 Antibodies among Pregnant Women in Port Harcourt, Rivers State, Nigeria. World Appl. Sci. J.2012; 16 (4): 589-598.

[3] Khayota Grace N. Prevalence of HSV-2, syphilis and HBV in HIV-1 individuals in selected health facilities in Nairobi, Kenya [MSc Thesis]. Kenyatta University, Kenya. 2012; 1-67.

[4] Okonko I, Anugweje K, Adeniji F, Abdulyekeen R. Syphilis, HIV, HCV and HBsAg co-infections among Sexually Active Adults. Nature and Science. 2012; 10(1):66-74.

[5] Pennap G, Akpu P, Adoga M. Treponema pallidum Infection among a Cohort of Pregnant Women in North Central Nigeria. Ame J of Trop Med \& Pub Health.2011; 1(2): 31-36.

[6] World Health Organization (2003): Guideline for the Management of Sexually Transmitted Infections. Revised version. World Health Organization, Geneva, Switzerland.

[7] Nicola M, Joseph E, Trevor P, Jeffrey D. Syphilis in the United States: An Update for Clinicians with an Emphasis on HIV Co-infection. Mayo Clin Proc. 2007; 82(9):1091-1102.

[8] Sia E, Jacqueline U, Akhtar H, Elizabeth M, Stig J, Noel E et al. Prevalence of sexually transmitted infections among pregnant women with known HIV status in northern Tanzania. Reproductive Health.2009; 6(4):1-8.

[9] Mulu A, Kassu A, Tessema B, Yismaw G, Tiruneh M, Moges F, et al. Sero-prevalence of syphilis and HIV-1 during pregnancy in a teaching hospital in northwest Ethiopia. Jpn J Infect Dis. 2007; 60:193-5.

[10] Eticha BT, Sisay Z, Alemayehu A, Shimelis T. Seroprevalence of syphilis among HIV-infected individuals in Addis Ababa, Ethiopia: a hospital-based cross-sectional study. BMJ Open. 2013; 3:e002293. Doi: 10.1136/bmjopen-2013-002566.

[11] Tessema B, Yismaw G, Kassu A, Amsalu A, Mulu A, Emmrich F, et al. Sero prevalence of HIV, HBV, HCV and syphilis infections among blood donors at Gondar University teaching hospital, northwest Ethiopia: declining trends over a period of five years. BMC Infect Dis. 2010; 10:111.9.

[12] Zenebe Y, Mulu W, Yimer M, Abera B. Sero-prevalence and risk factors of hepatitis B virus and human immunodeficiency virus infection among pregnant women in Bahir Dar city, northwest Ethiopia: a cross sectional study. BMC Infect Dis. $2014 ; 14: 118$.

[13] Buseri F, Seiyaboh E, Jeremiah Z. Surveying Infections among Pregnant Women in Niger Delta, Nigeria. J Glob Infect Dis.2010; 2(3):203-211.

[14] Mulu A, Kassu A, Tessema B, et al. Seroprevalence of syphilis and HIV-1 during pregnancy in a teaching hospital in northwest Ethiopia. Jpn J Infect Dis. 2007 Jul; 60(4):193-5.

[15] Global HIV/AIDS response. Epidemic update and health sector progress towards universal access. Geneva: World Health Organization; 2011. Available at: (http://www.afro.who.int/en/clusters-a-programmes/dpc/ acquired-immune-deficiency syndrome/features/3439-globalhivaidsresponse-progress-report-2011.html.).

[16] Gomez G, Kamb M, Newman L, Mark J, Broutet N, Hawkes S. Untreated maternal syphilis and adverse outcomes of pregnancy: a systematic review and meta-analysis. Bull World Health Organ. 2013; 91:217-26. doi:10.2471/BLT.12.107623.

[17] World Health Organization. Global Elimination of Syphilis: rationale and strategy for action. Geneva, Switzerland; 2007.

[18] Hawkes SJ, Gomez GB, Broutet N. Early antenatal care: does it make a difference to outcomes of pregnancy associated with syphilis? A systematic review and meta-analysis. PLoS-One. 2013; 8(2):e56713. Doi: 10.1371/journal. pone.0056713.

[19] World Health Organization: regional office for Africa. Analytical summary, 2010. Available at: http://www.aho.afro.who.int/profiles_information/index.php/ Ethiopia. 
72 Temesgen Anjulo Ageru and Temesgen Lera Abiso: Sero-Prevalence of HIV and Syphilis Infection Among Pregnant Mothers Attended Labor Ward at Wolaita Sodo University Teaching Referral Hospital: A Five-Year Retrospective Study (2012-2016)

[20] Health Sector Development Programme IV. Annual performance report. Addis Ababa, Government of Ethiopia: Ministry of Health; 2010.

[21] Ethiopian Health and Nutrition Research Institute. Report on the 2009 round antenatal care sentinel HIV surveillance in Ethiopia: Addis Ababa; 2011.

[22] Qin J, Yang T, Xiao S, Tan H, Feng T, Fu H. Reported estimates of adverse pregnancy outcomes among women with and without syphilis: a systematic review and meta-analysis. PLoS One. 2014; 9(7):e102203.

[23] Mwapasa V, Rogerson SJ, Kwiek JJ, et al. Maternal syphilis infection is associated with increased risk of mother-to-child transmission of HIV in Malawi. AIDS. 2006; 20(14):18691877.

[24] World Health Organization. Women and Health: Today's Evidence Tomorrow's Agenda. Geneva: Gender: World Health Organization; 2009. Available from: http://whqlibdoc.who.int/hq/2009/WHO_IER MHI_STM.09.1_eng.pdf.

[25] UNAIDS. Regional Fact Sheet 2012. Sub-Saharan Africa. Geneva: UNAIDS; 2012. Available from: http://www.unaids.org/sites/default/ files/en/media/unaids/contentassets/documents/epidemiology/ 2012/gr2012/2012_FS_regional_ssa_en.pdf

[26] Kendall T, Danel I. Research and Evaluation Agenda for HIV and Maternal Health in sub-Saharan Africa: Women and Health Initiative Working Paper No 1. Boston, MA: Women and Health Initiative, Harvard School of Public Health; 2014.

[27] Kebede B, Andargie A, Gebeyehu A. Birth outcome and correlates of low birth weight and preterm delivery among infants born to HIV-infected women in public hospitals of Northwest Ethiopia. Health. 2013; 5(7A4):25-34.

[28] Zeleke BM, Zelalem M, Mohammed N. Incidence and correlates of low birth weight at a referral hospital in Northwest Ethiopia. Pan Afr Med J. 2012; 12:4.

[29] Tricco AC, Antony J, Angeliki VA, et al. Safety and effectiveness of antiretroviral therapies for HIV-infected women and their infants and children: protocol for a systematic review and network meta-analysis. Syst Rev. 2014; $3: 51$.

[30] World Health Organization. Baseline Report on Global Sexually Transmitted Infection Surveillance 2012. Geneva; World Health Organization; 2013.

[31] Angelica M, Marta CA, Regina LN, Kelly RA, Anto N. Seroprevalence of HIV, HBV and Syphilis in Women at Their First Visit to Public Antenatal Clinics, Brazil. Sex Transm Dis. 2001; 28(12):710-713.

[32] Tiruneh M. Seroprevalence of multiple sexually transmitted infections among antenatal clinic attendees in Gonder Health Center, Northwest Ethiopia. Ethiop Med J. 2008; 32(4):359366.

[33] C. J. Uneke, D. D, Duhlinska and E. B. Igbinedion. Prevalence and Public-health Significance of HIV Infection and Anaemia among Pregnant Women Attending Antenatal Clinics in South-eastern Nigeria J HEALTH POPUL NUTR, 25(3), 2008, 328-335.

[34] Mbopi Kéou FX, Mbu R, Mauclère P, Andela A, Tetanye E,
Léké $\mathrm{R}$ et al. Antenatal HIV prevalence in Yaounde, Cameroon. Int J STD AIDS. 1998; 9(7):400-4002.

[35] L. E Abongwa, A. M Clara, NA. Edouard and N. H. Ngum. Sero-Prevalence of Human Immunodeficiency Virus (HIV) and Hepatitis B Virus (HBV) Co-Infection among Pregnant Women Residing in Bamenda Health District, Cameroon. Int. J. Curr. Microbiol. App. Sci (2015) 4(12): 473-483.

[36] Yusuf K, Julius M, Raphael I, Julius M, Raphael I, John C et al. Trends in HIV \& syphilis prevalence and correlates of HIV infection: results from cross-sectional surveys.

[37] Robert L, Silvia M, Lee H, Renaldo I, Roberta B, Sonia B et al. Prevalence of Sexually Transmitted Diseases in Young Women Seeking HIV Testing in Rio de Janeiro, Brazil. Sex Transm Dis. 2004; 31(1): 67-72.

[38] Thu-Ha D, Mary L, Veerle M. Integration of Preventing Mother-To-Child Transmission of HIV and Syphilis Testing and Treatment in Antenatal Care Services in the Northern Cape and Gauteng Provinces, South Africa. Sex Transm Dis. 2013; 40(11):846-850.

[39] Federal Ministry of Heath/Ethiopian Health and Nutrition Research Institute. Report on the 2009 Round Antenatal Care Sentinel HIV Surveillance in Ethiopia. Addis Ababa, Ethiopia; 2011.

[40] Henok S. Prevalence of HIV among antenatal care attendants at Kazanchis Health center in the last five years (2006-2010), Addis Ababa, Ethiopia. [MSc Thesis]. Addis Ababa University, Ethiopia. 2011; 1-38.

[41] Tiruneh M. Seroprevalence of multiple sexually transmitted infections among antenatal clinic attendees in Gonder Health Center, Northwest Ethiopia. Ethiop Med J. 2008; 46(4):359366.

[42] Endris M, Deressa D, Belyhun $Y$ and Moges F. Seroprevalence of syphilis and human immunodeficiency virus infections among pregnant women who attend the University of Gondar teaching hospital, Northwest Ethiopia: a cross sectional study. BMC Infectious Diseases (2015) 15:111.

[43] Melku M. Kebede A, Addis Z. Magnitude of HIV and syphilis Seroprevalence among pregnant women in Gondar, northwest Ethiopia: a cross-sectional study. HIV/AIDS - Research and Palliative Care 2015:7: 175-182

[44] Krunal D. Mehta, Sejul Antala, Madhulika Mistry, Yogesh Goswami. Seropositivity of hepatitis B, hepatitis C, syphilis, and HIV in antenatal women in India. J Infect Dev Ctries 2013; 7(11):832-837.

[45] José M, Sofia B, Francisco R, Juan C, Gloria R, Félix G. Seroprevalence of HIV-1, HBV, HTLV-1 and T. pallidum infections among pregnant women in a rural hospital in Southern Ethiopia. Journal of clinical virology.2011; 51(1):83-85.

[46] Kuznik A, Lamorde M, Nyabigambo A, Manabe YC. Antenatal syphilis screening using point-of-care testing in subSaharan African countries: a cost-effectiveness analysis. PLoS Med. 2013; 10(11):e1001545. doi:10.1371/journal.pmed.1001545.

[47] Frickmann H, Schwarz NG, Girmann M, et al. Serological survey of HIV and syphilis in pregnant women in Madagascar. Trop Med Int Health. 2013; 18(1):35-39. 
[48] Nessa A, Islam MN, Ashraf F, et al. Seroprevalence of Treponema Pallidum antibody among pregnant population of Bangladesh. J Obstet Gynaecol Res. 2011; 37(11):1625-1630.

[49] Kebede E and Chamiso B. Prevalence of syphilis in pregnancy in Addis Ababa. East African Medical Journal. 2000; 77(4):212-215.

[50] Yahya-Malima KI, Evjen-Olsen B, Matee MI, Fylkesnes K, Haarr L. HIV-1, HSV-2 and syphilis among pregnant women in a rural area of Tanzania: prevalence and risk factors. $B M C$ Infect Dis. 2008; 8:75.
[51] Azeze B, Fantahun M, Kidan KG, Haile T. Seroprevalence of syphilis amongst pregnant women attending antenatal clinics in a rural hospital in north west Ethiopia. Genitourin Med. 1995; 71(6):347-350.

[52] Ojo DA, Oyetunji IAO. Sero-prevalence of syphilis among pregnant women In Osogbo in Southwestern Nigeria. ASSET Series B. 2007; 6(1): 61-65.

[53] Omisakin CT, Esan AJ, Fasskin KA et.al. Syphilis and HIV co-infection among pregnant women in Nigeria: Prevalence and trend. ISRR 2014(2):95-100. 